$>$ À la suite de l'accident de Tchernobyl, les énormes quantités d'isotopes radioactifs libérées dans l'atmosphère ont fortement contaminé les populations environnantes, ce d'autant qu'aucune mesure de protection n'a été prise rapidement. Les doses d'irradiation les plus importantes ont été délivrées par les iodes radioactifs à la thyroïde, et la seule conséquence sanitaire directement liée à l'irradiation au niveau des populations contaminées est l'augmentation de l'incidence des cancers de la thyroïde chez les sujets qui étaient enfants en 1986 et qui vivaient en Biélorussie, en Ukraine ou en Russie. <
Accident de centrale nucléaire et risque de cancer de la thyroïde

\section{Les conséquences} sanitaires de Tchernobyl

Martin Schlumberger ${ }^{1}$, Bernard Le Guen ${ }^{2}$

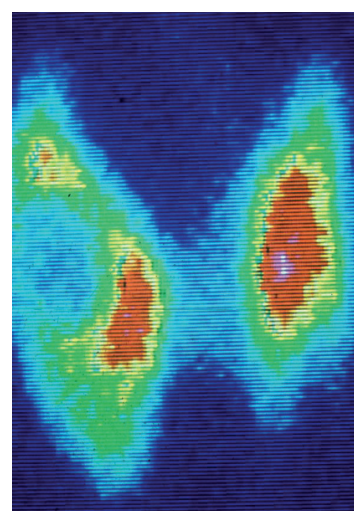

${ }^{1}$ Université Paris-Sud, service de médecine nucléaire et cancérologie endocrinienne, Institut Gustave Roussy, 94805 Villejuif Cedex, France ; ${ }^{2} \varepsilon D F$ (Électricité de France), Direction production ingénierie, division production nucléaire, 93282 Saint-Denis Cedex, France. martin.schlumberger@igr.fr
Lors de l'accident de Tchernobyl le 26 avril 1986, les énormes quantités de produits radioactifs libérés dans l'atmosphère pendant une dizaine de jours ont contaminé des régions de Biélorussie, d'Ukraine et de Russie (Tableau I). Une augmentation considérable de l'incidence des cancers de la thyroïde a été observée par la suite chez les personnes vivant dans ces régions et qui étaient âgées de moins de 18 ans au moment de l'accident.

Vingt-six ans après cet accident, un bilan peut être établi qui permet de guider l'information et la protection des populations $[1,2]$.

\section{L'accident de Tchernobyl en 1986}

À la suite d'erreurs humaines, la cuve d'un réacteur de la centrale a explosé, et les barres de graphite ont pris feu, projetant à plusieurs kilomètres d'altitude des quantités considérables de radioactivité (Tableau I et Encadré). Le panache radioactif a contaminé les régions avoisinantes du sud de la Biélorussie, du nord de l'Ukraine et des régions voisines de la Russie [1], puis s'est déplacé vers la Pologne, les pays scandinaves et l'ouest de l'Europe.

Photo : scintigraphie de la thyroïde. L'iode radioactif injecté est concentré par la thyroïde. Les deux lobes sont visualisés et le nodule palpé du lobe droit ne fixe pas l'iode radioactif, il s'agit d'un nodule froid (৫ Martin Schlumberger).
Les conséquences liées à l'exposition aux rayonnements ionisants ont pour origine soit une irradiation de l'organisme par une exposition externe à des sources radioactives de proximité, soit une contamination par des substances radioactives. Celle-ci est externe en cas de dépôt sur la peau, interne en cas d'ingestion (denrées contaminées) ou d'inhalation (gaz et aérosols radioactifs). Les conséquences potentielles de cette exposition peuvent être précoces et de gravité proportionnelle à la dose reçue (en cas de fortes doses reçues par les intervenants sur la centrale), ou retardées et leur risque de survenue chez les intervenants et les populations contaminées sera fonction de la dose reçue.

\section{Les principaux radioisotopes impliqués}

L'iode 131 et le césium 137 libérés en grandes quantités sont les isotopes radioactifs les plus préoccupants d'un point de vue sanitaire (Tableau 1). D'autres radionucléides rejetés jouent un rôle plus ou moins important, notamment les gaz rares (par exposition externe), l'iode 132 et 133 et le césium 134 (dans ce cas, la contamination se fait par voie interne). Le césium 137, dont la demi-vie est de 30 ans, peut être responsable d'une contamination de l'environnement qui peut durer des décennies. II s'intègre dans la chaîne alimentaire et peut ainsi contaminer les individus. Dans l'organisme, le césium a le même comportement que le potassium et se répartit dans tous les tissus, sans être concentré dans un tissu particulier. La dose cumulée au corps entier a été inférieure à $50 \mathrm{mSv}$ pendant les 10 ans qui ont suivi l'accident de Tchernobyl pour les populations les plus contaminées de Biélorussie, d'Ukraine et de Russie [1]. Les isotopes radioactifs de l'iode ont une demi-vie brève, quelques heures pour les iodes 132 et 


\begin{tabular}{lcc}
\hline & Rejets de Tchernobyl & $\begin{array}{c}\text { Rejets de } \\
\text { Fukushima }\end{array}$ \\
\hline lode 131 & $1800000 \mathrm{TBq}$ & $130000 \mathrm{TBq}$ \\
\hline Césium 137 & $85000 \mathrm{TBq}$ & $11000 \mathrm{TBq}$ \\
\hline Rejets totaux & $5200000 \mathrm{TBq}$ & $630000 \mathrm{TBq}$ \\
\hline
\end{tabular}

Tableau I. Importance comparée des rejets d'iode 131 et de césium 137 lors des accidents de Tchernobyl et de Fukushima.

Les quantités totales de produits radioactifs rejetés fin août 2011 ont été fournies par l'opérateur japonais TEPCO. TBq = terabecquerel $\left(10^{12} \mathrm{~Bq}\right)$.

133, et 8 jours pour l'iode 131. Ils peuvent contaminer les individus par inhalation mais surtout par ingestion d'aliments frais contaminés produits sur place (lait et légumes). Ils sont alors concentrés par la thyroïde qui va ainsi recevoir une dose de radiations 1000 à 10000 fois supérieure à la dose reçue par les autres organes [3] (Tableaux II et III). Le risque disparaît au bout de quelques semaines.

\section{Bilan sanitaire des différents types de populations exposées}

Le bilan sanitaire concerne les 600 travailleurs et pompiers de la centrale, les 1125 pilotes et membres d'équipages d'hélicoptères, les 530000 liquidateurs intervenus entre 1987 et 1990 pour la construction du sarcophage, les 130000 personnes qui vivaient dans un rayon de $30 \mathrm{~km}$ autour de la centrale et qui ont été évacuées, et les populations - dont 2 millions d'enfants - qui vivaient en 1986 dans les régions fortement contaminées de Biélorussie, d'Ukraine et de Russie.

Parmi les 600 travailleurs de la centrale, 237 ont été hospitalisés dont 134 pour un syndrome d'irradiation aiguë avec dépression médullaire, dermatite aiguë et complications gastro-intestinales. Ces sujets ont reçu des doses « corps entier » comprises entre 1 et $16 \mathrm{~Sv}$. Parmi les 134 sujets atteints d'un syndrome d'irradiation aiguë, 13 ont eu une greffe de moelle, parmi lesquels deux seulement ont survécu. Au total, 47 décès sont survenus, 28 pendant l'année qui a suivi l'accident, 19 entre 1987 et 2006. Les causes des décès survenus après la première année sont mal déterminées. Les doses au niveau de la peau ont été 10 à 30 fois plus élevées que les doses au niveau de la moelle osseuse ; c'est la conséquence de l'irradiation des émetteurs $\beta$ déposés sur la peau, faute d'une protection suffisante. Les brûlures cutanées ont été sévères, mais aucun cancer sur brûlures n'a été observé.

L'excès de cataractes a été confirmé chez les travailleurs de la centrale et dans le groupe des liquidateurs. Cette conséquence connue de l'irradiation du cristallin est également suspectée dans la population générale jeune, bien que d'autres causes puissent être évoquées [1]. Chez les liquidateurs, les données épidémiologiques sont peu fiables, et probablement biaisées. La dose individuelle reçue, mesurée chez $50 \%$ d'entre eux, a été en moyenne de 117 mSv [1]. Certaines études ont rapporté un faible excès de leucémies et de cancers du sein pour des doses supérieures à $200 \mathrm{mSv}$, ce qui est en accord avec l'excès de cancers observé chez les survivants des explosions atomiques d'Hiroshima et Nagasaki [4].

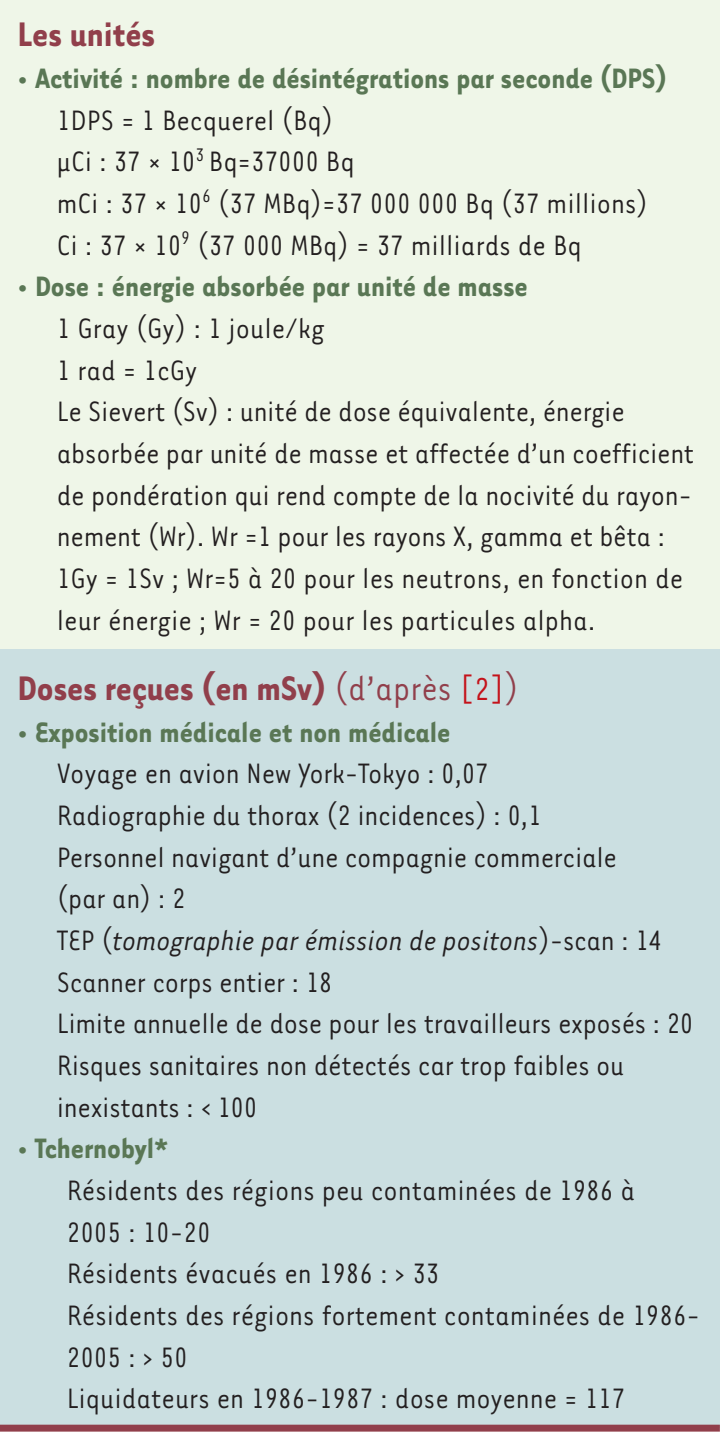

Tableau II. Unités et doses délivrées.

* La dose cumulée sur la durée indiquée est à rajouter à la dose naturelle annuelle (2,4 mSv/an en France).

Pendant les semaines et les mois qui ont suivi l'accident, des craintes ont été formulées concernant la survenue possible de leucémies chez les populations contaminées et d'anomalies dans leur descendance. Ceci n'a pas été confirmé par la suite, ce qui est en accord avec les doses reçues par ces populations : pendant les dix années qui ont suivi l'accident, les doses cumulées « corps entier » ont été au maximum d'environ $50 \mathrm{mSv}$ dans les régions les plus contaminées, et inférieures à $10 \mathrm{mSv}$ dans les autres régions. Rappelons qu'en France, la dose moyenne annuelle reçue par chaque habitant est de 2 à $3 \mathrm{mSv}$ (Tableau II). Les craintes concernant les effets de l'irradiation du fœtus chez les femmes qui étaient enceintes en 1986 ont induit un nombre important d'interruptions de grossesse, mais aucune 


\section{Origines techniques des risques associés aux centrales nucléaires}

La conception d'un réacteur à eau sous pression (PWR) en France est bien différente de celle des réacteurs de Tchernobyl, d'une part, et de Fukushima, d'autre part, et la sûreté de l'EPR (réacteur pressurisé européen) a encore été améliorée. Dans un réacteur PWR de 1300 MW, la radioactivité des iodes, produits de fission, est d'environ $3,10^{19} \mathrm{~Bq}$. Les iodes étant volatiles, ils risquent de se retrouver dans l'environnement lors d'un accident grave.

En l'absence prolongée de refroidissement, les gaines de combustible nucléaire s'échauffent au-delà de $1200^{\circ} \mathrm{C}$ et libèrent, par réaction chimique, de l'hydrogène. L'hydrogène est capté par des pièges passifs appelés recombineurs d'hydrogène, ce qui évite le risque d'explosion. Le cœur, constitué de l'ensemble des éléments combustibles, se met à fondre et peut traverser la cuve du réacteur. Cet amas de combustibles et de métaux divers s'appelle le corium, et peut se retrouver au fond du bâtiment réacteur, appelé enceinte de confinement (de la radioactivité). Si le combustible, a fortiori le corium, n'est pas refroidi, les centaines de $\mathrm{m}^{3}$ d'eau contenues dans le bâtiment se transforment en vapeur d'eau et la pression augmente dans le bâtiment réacteur. Les systèmes de contrôle évitent d'atteindre la limite de pression de l'enceinte de confinement ( 5,5 bar), ce qui peut nécessiter de la décomprimer par un rejet dans l'atmosphère à travers un filtre à sable qui est présent sur l'ensemble des centrales nucléaires actuelles. Ce filtre à sable permet de limiter la contamination radioactive de l'environnement, notamment par le césium 137. L'EPR dispose de systèmes de refroidissement et d'aspersion, mais également d'un circuit d'évacuation ultime ( $(V U)$ de la chaleur contenue à l'intérieur du bâtiment. Ses principales fonctions sont le refroidissement du corium par de l'eau qui vise à le submerger, évitant ainsi une augmentation importante de la pression, et à limiter la quantité d'iode radioactif en suspension dans l'atmosphère de l'enceinte de confinement par l'injection de la soude. L'ensemble des dispositions mises en place sur l'EPR, qui permettent d'obtenir une géométrie refroidissable du corium (récupération et étalement du corium), puis un refroidissement par $\varepsilon V U$, justifie l'absence de filtre à sable. Notons que dans les conditions normales de fonctionnement des centrales nucléaires, il n'y a pas d'augmentation de l'incidence des cancers et des leucémies chez les populations qui habitent à proximité des centrales. L'excès de leucémies aiguës lymphoblastiques rapporté récemment chez les enfants vivant à proximité des centrales nucléaires est crédible. Il existe toutefois une forte incertitude sur la cause de ces leucémies, et cet excès est limité dans le temps et l'espace. II s'agit d'un phénomène rare, noté dans seulement 3 des 198 études effectuées sur des sites industriels nucléaires dans 10 pays, qui a été observé dans d'autres sites industriels non nucléaires et qui est sans rapport avec la dose d'irradiation délivrée aux populations (de l'ordre du microSv) ni avec la proximité des centrales nucléaires [15].

augmentation des anomalies (mortinatalité, malformation, anomalie génétique) n'a été observée car elles apparaissent pour des niveaux de doses supérieurs à 100 mSv. Depuis l'accident, il n'y a pas d'augmentation de la fréquence des anomalies héréditaires chez les enfants nés de sujets contaminés lors de l'accident, ce qui est en accord avec les études effectuées chez les descendants de sujets traités par radiothérapie pour cancer de l'enfance ou par l'iode 131 pour cancer de la thyroïde $[5,6]$.

\begin{tabular}{|lccc|}
\hline $\begin{array}{c}\text { Doses } \\
\text { reçues par } \\
\text { les organes } \\
\text { (mGy) }\end{array}$ & $\begin{array}{c}\text { Fixation de l'iode 131 } \\
\text { par la thyroíde à 24 h }\end{array}$ \\
$\begin{array}{l}\text { (\% de l'activité d'iode } \mathbf{1 3 1} \\
\text { administrée) }\end{array}$ \\
\hline Thyroïde & 2800 & 8000 & 13000 \\
\hline Gonades & 1,0 & 1,0 & 1,0 \\
\hline Moelle osseuse & 1,4 & 2,0 & 2,6 \\
\hline Estomac & 17 & 16 & 14 \\
\hline Corps entier & 2,4 & 4,7 & 7,1 \\
\hline
\end{tabular}

Tableau III. Doses (mGy ou mSv) délivrées après l'administration d'une activité de $1 \mathrm{mCi}$ (soit $37 \mathrm{MBq}$ d'iode 131) en fonction du pourcentage d'iode 131 fixé par la thyroïde à $24 h$. L'iode 131 est concentré par la thyroïde et la dose délivrée à la thyroïde est 1000 à 10000 fois plus importante que celle délivrée aux autres organes [3]. En cas de contamination, la thyroïde doit être protégée par la prise d'iodure de potassium (KI).

En revanche, les conséquences psychologiques de l'accident ont été majeures. Elles sont liées à l'irradiation, mais aussi à l'absence de prophylaxie, à des erreurs de communication, au déplacement des populations, et à la dégradation des conditions économiques - avec recrudescence de l'alcoolisme et du tabagisme - et des conditions sanitaires. Elles sont responsables de l'augmentation observée des taux de morbidité et de mortalité, qui n'est donc pas liée directement à l'exposition aux rayonnements [1].

En conclusion, vingt-six ans après, le suivi de ces différentes populations ne révèle pas d'excès significatif de leucémies ni de cancers autres que les cancers de la thyroïde, survenus chez les sujets exposés à un âge inférieur à 18 ans. Les pathologies thyroïdiennes sont liées à la contamination par les iodes radioactifs qui sont concentrés dans la thyroïde qui, de ce fait, est irradiée (Tableau III). La dose reçue par la thyroïde des enfants a atteint plusieurs centaines de $\mathrm{mSv}$ dans certaines régions de Biélorussie, d'Ukraine et de Russie. Les pathologies thyroïdiennes survenues depuis sont détaillées plus loin.

\section{Accident de Tchernobyl : les conséquences sur la glande thyroïde}

\section{Conséquences sur la population d'enfants et d'adolescents}

Environ deux millions d'enfants et d'adolescents âgés de moins de 18 ans vivaient en 1986 dans des régions 
fortement contaminées de Biélorussie, d'Ukraine et de Russie. Les premiers cas de cancer de la thyroïde ont été observés en 1990 chez des enfants de moins de 14 ans. Depuis, environ 7000 personnes âgées de moins de 18 ans lors de l'accident ont développé un cancer thyroïdien. Son incidence actuelle reste élevée et ne diminue pas. Les enfants ont un risque d'autant plus élevé qu'ils étaient plus jeunes lors de l'accident (Tableau IV) et, pour chaque tranche d'âge, le risque augmente avec la dose d'irradiation délivrée à la thyroïde. L'augmentation du risque est identique chez les filles et chez les garçons. Ce risque est important chez les personnes contaminées peu après la naissance ou in utero, mais n'est pas augmenté chez les personnes nées plus d'un an après l'accident, alors qu'il n'existait plus de risque de contamination par les iodes radioactifs.

La majorité des cancers de la thyroïde survenus chez les enfants et les adolescents pendant les premières années après l'accident sont imputables à l'accident car, à cet âge, l'incidence du cancer de la thyroïde spontané est très faible ( 1 cas/million d'enfants). De plus, ces jeunes enfants ont souvent développé des tumeurs volumineuses et cliniquement évidentes (Tableau IV). En revanche, un meilleur dépistage peut rendre compte d'une partie de l'augmentation des cancers de la thyroïde observés par la suite, car l'incidence des cancers non radio-induits augmente avec l'âge, même chez les personnes jeunes, l'âge médian de survenue d'un cancer de la thyroïde non radio-induit étant de 45 ans [1].

\section{Conséquences sur la population d'adultes et de personnes âgées}

Chez les personnes qui étaient adultes en 1986 et vivaient en Biélorussie, en Ukraine ou en Russie, ainsi que chez les «liquidateurs » qui ont travaillé sur le site de Tchernobyl, une augmentation de l'incidence des petits cancers de la thyroïde a été mise en évidence, mais dans des proportions beaucoup moins importantes que chez les enfants, et elle n'est pas liée à la dose reçue par la thyroïde. De fait, cette

\begin{tabular}{|ccccc|}
\hline $\begin{array}{c}\text { Âge en } \\
\mathbf{1 9 8 6}\end{array}$ & $\begin{array}{c}\mathbf{1 9 7 1 -} \\
\mathbf{1 9 8 5}\end{array}$ & $\begin{array}{c}\mathbf{1 9 8 6 -} \\
\mathbf{2 0 0 0}\end{array}$ & $\begin{array}{c}\text { Facteur } \\
\text { de RR }\end{array}$ & $\begin{array}{c}\text { Sex ratio } \\
\text { (F/M) }\end{array}$ \\
\hline $0-14$ & 8 & 703 & 87,8 & 1,6 \\
\hline $15-18$ & 21 & 267 & 12,7 & 1,8 \\
\hline 19 & 1465 & 6719 & 4,5 & 5,2 \\
\hline $\begin{array}{l}\text { Ensemble de } \\
\text { la population }\end{array}$ & 1494 & 7689 & 5,1 & \\
\hline
\end{tabular}

Tableau IV. Incidence du cancer de la thyroïde en Biélorussie après l'accident de Tchernobyl.

La période analysée (1986-2000) a été comparée à la période 1971-1985 en fonction de l'âge en 1986. Le risque est fonction de l'âge lors de l'exposition : il est maximal chez les sujets qui avaient moins de 14 ans en 1986 et diminue avec l'âge. L'augmentation de l'incidence observée pour les sujets de plus de 19 ans dans la période 1986-2000 peut être liée à un meilleur dépistage. Les chiffres indiquent le nombre total de cas pour la période indiquée. augmentation a été attribuée principalement à un meilleur dépistage [1].

\section{Incidence des cancers thyroïdiens dans les régions limitrophes de Tchernobyl}

La réalité de cette augmentation de l'incidence des cancers de la thyroïde observée chez les personnes âgées de moins de 18 ans lors de l'accident et, parmi elles, essentiellement chez les enfants âgés de moins de 10 ans, ainsi que ses causes, ont été l'objet de controverses pendant plusieurs années.

Il était démontré que l'exposition aux radiations ionisantes par voie externe dans le cadre de traitements par radiothérapie pour une pathologie maligne ou bénigne survenue pendant l'enfance, par exemple pour un cancer de la tête et du cou ou pour une maladie de Hodgkin, augmente le risque de cancer de la thyroïde. Toutefois, des études suédoises menées chez les sujets exposés à l'iode 131 pour des raisons médicales (scintigraphie de la thyroïde ou traitement d'une hyperthyroïdie) n'avaient pas montré d'augmentation du risque de cancer de la thyroïde, et ceci avait été attribué au faible débit de dose de l'iode 131, dont la dose totale est délivrée en plusieurs jours alors qu'elle est délivrée en quelques minutes lors de la radiothérapie externe. En fait, la majorité des sujets suédois étaient adultes lors de l'exposition à l'iode 131, âge auquel la thyroïde est peu ou pas sensible à l'effet cancérigène des radiations ionisantes, comme l'ont montré les études après irradiation par voie externe. Ces observations ne permettent donc pas d'exclure l'effet cancérigène de l'iode 131 sur la thyroïde de l'enfant et de l'adolescent [7-11]. Cette mauvaise interprétation des données suédoises est une des raisons qui expliquent l'absence de mesures prophylactiques claires prises par les autorités soviétiques dans les premières semaines qui ont suivi l'accident.

D'autre part, la rapidité de survenue des cancers de la thyroïde après l'accident a surpris car, dans les observations antérieures, l'augmentation de l'incidence de ces cancers après irradiation externe ne survenait que plus de 5 à 10 ans après l'exposition. Plusieurs cas de cancers de la thyroïde ont en effet été observés dès 1990 parmi le nombre important d'enfants contaminés qui étaient âgés de moins de 14 ans (la plupart ayant d'ailleurs moins de 10 ans) au moment de l'accident. Actuellement, il existe un consensus scientifique général pour attribuer cette augmentation d'incidence à la contamination par les iodes radioactifs, et notamment par l'iode 131. La contamination a été provoquée par l'inhalation et la consommation de produits frais 
contaminés produits sur place et, notamment, celle du lait frais. Aucune mesure de protection des populations n'avait alors été prise, ni aucune consigne de confinement. Par exemple, les cérémonies du $1^{\text {er }}$ mai 1986 en Biélorussie, qui se déroulaient en plein air et auxquelles assistaient de nombreux enfants, ont été maintenues. De même, aucune restriction alimentaire et aucune surveillance ni des fourrages pour le bétail ni du lait produit sur place n'ont été instituées. Par ailleurs, les populations qui vivaient autour de la centrale n'ont été évacuées que plusieurs jours après l'accident. Le risque de cancer de la thyroïde a été encore majoré par l'état de carence iodée des populations présentes dans de nombreuses régions fortement contaminées. Lors de l'accident, cette carence a augmenté la fixation de l'iode radioactif dans la thyroïde, donc la dose d'irradiation délivrée et, ultérieurement, elle a stimulé la multiplication des cellules thyroïdiennes. Au contraire, le risque de cancer de la thyroïde a été diminué par l'iode stable qui a été administré à certaines populations, même à distance de la contamination.

Les doses reçues par la thyroïde des enfants qui vivaient dans les régions les plus contaminées de Biélorussie, d’Ukraine et de Russie ont ainsi pu atteindre plusieurs centaines de mSv. La dose a été plus élevée chez les jeunes enfants dont la thyroïde est certes plus petite (poids de $1 \mathrm{~g}$ à 1 an versus 15 à $20 \mathrm{~g}$ à l'âge adulte) mais qui concentre autant d'iode 131 qu'à un âge plus avancé, et pour une fixation donnée, la dose qui est fonction de la masse de la thyroïde sera beaucoup plus importante chez le jeune enfant. L'estimation des doses individuelles délivrées à la thyroïde a été effectuée à distance de l'accident et est donc peu précise, sauf pour deux cohortes de sujets (12 000 en Biélorussie et 13000 en Ukraine), chez lesquels la contamination a été mesurée directement par comptage de l'activité thyroïdienne et urinaire dans les semaines qui ont suivi l'accident. Ces données confirment celles des études antérieures faites dans les cohortes de patients ayant eu une irradiation externe thérapeutique : pas d'effet décelable pour des doses à la thyroïde $<100 \mathrm{mGy}$, et excès de risque relatif par gray $(\varepsilon \varepsilon R / G y)$ de cancer de la thyroïde après contamination interne par les iodes radioactifs - 3,16 à 7,35 selon les études - comparable à celui observé après irradiation externe ( $\varepsilon R R / G y=7,7)[8]$.

Ces cancers de la thyroïde représentent la seule anomalie organique décelée dans les populations contaminées qui soit directement imputable à la contamination radioactive liée à l'accident.

\section{Incidence de l'accident sur les cancers thyroïdiens en Pologne et en France}

En dehors des régions limitrophes d'Ukraine, de Biélorussie et de Russie, aucune augmentation des cancers de la thyroïde n'a été attribuée à la contamination liée à l'accident de Tchernobyl. En Pologne, 18 millions de doses d'iode stable ont été distribuées les jours suivant l'accident, et la dose d'irradiation de la thyroïde est restée bien inférieure à $50 \mathrm{mSv}$. Depuis, aucune augmentation de l'incidence du cancer de la thyroïde liée à cette contamination n'a été décelée [12]. L'augmentation de l'incidence du cancer de la thyroïde observée dans la plupart des pays industrialisés, dont la France [13], depuis les années 1970 est attribuée essentiellement à un meilleur dépistage des petits cancers de la thyroïde par échographie, cytoponction et une meilleure pratique médicale, ainsi que, probablement, à d'autres facteurs tels qu'une augmentation de la fréquence de l'obésité [14]. En effet, la majorité des petits cancers de la thyroïde peuvent rester méconnus sans progresser, et ne sont découverts que lorsqu'ils sont recherchés. En France, la dose délivrée à la thyroïde des enfants à la suite de l'accident de Tchernobyl a été au maximum de quelques mSv dans l'est du pays, soit de l'ordre de grandeur de l'irradiation naturelle annuelle (2,4 mSv, voir Tableau II). Dans les années qui ont suivi l'accident, les registres n'ont décelé aucune augmentation de l'incidence des cancers de la thyroïde chez l'enfant. Par ailleurs, la tendance temporelle à l'augmentation de l'incidence du cancer thyroïdien a été plus importante dans l'ouest de la France - région sans aucune retombée radioactive - que dans l'est du pays. La mise en évidence de plusieurs cas de pathologies thyroïdiennes fonctionnelles ou morphologiques dans certaines populations peut s'expliquer simplement par un meilleur dépistage.

\section{La prophylaxie des conséquences d'un accident nucléaire}

\section{Prophylaxie par l'iodure de potassium}

Les conséquences de l'accident de Tchernobyl montrent l'importance de la prophylaxie de l'irradiation de la thyroïde lors d'une contamination atmosphérique par les iodes radioactifs, et ceci est possible par une contre-mesure simple et efficace : l'administration d'iode stable en quantité suffisante pour saturer la thyroïde et ainsi l'empêcher de concentrer les iodes radioactifs. La dose de radiations délivrée à la thyroïde par les iodes radioactifs dépend de la concentration de radioactivité dans la thyroïde (rapport entre l'activité fixée et la masse de la thyroïde) et du temps de séjour de l'iode radioactif dans la thyroïde (Tableau III). Pour une contamination donnée, la dose sera d'autant plus importante que la fixation est élevée et que la masse de la thyroïde est faible. L'iode stable administré sous la forme d'iodure de potassium (KI) inhibe la fixation des iodes radioactifs et peut réduire de plus de $95 \%$ l'irradiation de la thyroïde, s'il est pris en quantités suffisantes et quelques heures avant la contamination, ou si la prise est faite simultanément à la contamination, et de $50 \%$ seulement si elle a lieu 6 h après (Tableau V) [16]. La diminution de la fixation de l'iode 131 persiste pendant 48-72 h, puis la fixation réapparaît et ceci a deux conséquences : d'une part, il est inutile de prendre l'iode stable en l'absence de contamination, avant que sa prise soit conseillée par les pouvoirs publics; 


\begin{tabular}{|c|c|c|}
\hline $\begin{array}{c}\text { Temps entre } \\
\text { l'administration } \\
\text { du KI } \\
\text { et l'exposition à } \\
\text { l'iode radioactif } \\
\text { (h) }\end{array}$ & $\begin{array}{c}\text { Dose } \\
\text { à la thyroïde } \\
(\mathrm{mSv} / \mathrm{MBq})\end{array}$ & $\begin{array}{c}\text { Degré } \\
\text { de protection } \\
\text { de la thyroïde } \\
\text { (\%) }\end{array}$ \\
\hline$-96 h$ & 375 & 5 \\
\hline$-72 \mathrm{~h}$ & 267 & 32 \\
\hline$-48 h$ & 97 & 75 \\
\hline$-24 h$ & 25 & 93 \\
\hline $\mathrm{Oh}$ & 12 & 97 \\
\hline$+2 h$ & 81 & 80 \\
\hline$+8 h$ & 235 & 40 \\
\hline$+16 \mathrm{~h}$ & 329 & 17 \\
\hline$+24 h$ & 367 & 7 \\
\hline
\end{tabular}

Tableau V. Efficacité de l'iodure de potassium (KI) pour protéger la thyroïde après l'administration d'une activité de $1 \mathrm{MBq}$ d'iode 131 .

La dose utilisée est de $130 \mathrm{mg}$ de KI, soit 100 mg d'iodure (d'après [13]).

et d'autre part, la prise d'iode stable doit être répétée en cas de contamination persistant au-delà de quelques jours. Cette protection n'est efficace que contre les seuls isotopes radioactifs de l'iode et non contre l'ensemble des radioéléments susceptibles d'être rejetés; elle doit donc s'inscrire dans une prévention plus large qui inclue la mise à l'abri, les restrictions alimentaires, voire l'évacuation des populations selon l'importance de la contamination.

\section{Organisation et méthodologie du prétraitement par l'iodure de potassium}

En raison de l'importance d'une prise précoce d'iode stable, les pouvoirs publics ont, depuis 1997, chargé Électricité de France (EDF) d'en assurer la distribution à l'ensemble de la population vivant dans un rayon de $10 \mathrm{~km}$ autour des 19 sites nucléaires français. Ceci assure une disponibilité permanente des comprimés d'iode. L'efficacité de cette prédistribution est régulièrement testée, en sensibilisant les populations riveraines à l'occasion d'exercices locaux de crise $[17,18]$. Pour compléter cette distribution, dans le cadre d'un plan BIOTOX, des stocks de comprimés d'iode stable ont été constitués dans tous les départements où existent des installations nucléaires, ainsi que dans les départements limitrophes. Chaque comprimé de KI est dosé à $65 \mathrm{mg}$ - soit $50 \mathrm{mg}$ d'iodure - et contient aussi des excipients (cellulose microcristalline, huile de coton hydrogénée, silice colloïdale anhydre). Les comprimés sont quadrisécables et solubles dans l'eau, le lait et les jus de fruits, et sont présentés en boîtes de 10 comprimés sous blister. II s'agit d'un médicament fabriqué par la Pharmacie centrale des armées (PCA), dont I'AMM (autorisation de mise sur le marché) date du 24 février 2009, et que toute personne en France peut se procurer.
Quatre campagnes nationales de distribution ont été organisées, et la périodicité du renouvellement des comprimés d'iode a été portée de trois à cinq, puis à sept ans. Lors de la dernière campagne de distribution (2009-2010), la population riveraine de chaque site nucléaire a été dans un premier temps sensibilisée, via les pharmacies, à la prochaine réception d'un courrier nominatif par voie postale. Ce courrier invitait toutes les personnes à venir retirer gratuitement les boîtes de comprimés de KI en pharmacie, ainsi que des documents concernant, d'une part, les éventuels effets indésirables de l'iode et, d'autre part, le contrôle et la sûreté de la centrale nucléaire concernée. D'après les bons de retrait nominatifs remis au pharmacien en échange de la boîte de $\mathrm{KI}$, les comprimés ont été distribués à plus de $60 \%$ de la population concernée. Un envoi postal complémentaire a permis d'assurer une couverture totale de la population. Au total, 530000 boîtes de KI (contenant chacune 10 comprimés) ont été distribuées. De plus, des stocks de comprimés de KI ont été constitués dans toutes les communautés : crèches, écoles, centres de vacances, entreprises, etc.

Les méthodes actuellement disponibles (compteur Geiger, par exemple) peuvent détecter de très faibles contaminations radioactives qui, lorsqu'elles ne sont pas associées à un risque sanitaire, ne justifient aucune mesure prophylactique. En cas d'accident, l'ordre d'administration du KI est donné par les autorités compétentes (préfet), le niveau d'intervention retenu étant le risque d'une exposition de la thyroïde des enfants à une dose de $50 \mathrm{mSv}$.

Les quantités d'iode à administrer sont : (1) pour les nourrissons : $16 \mathrm{mg}$ de $\mathrm{KI}$, soit $1 / 4$ de comprimé en 1 fois ; (2) pour les enfants jusqu'à 3 ans : $32,5 \mathrm{mg}$, soit $1 \frac{2}{2}$ comprimé ; 3 ) pour les enfants de 3 à 12 ans : $65 \mathrm{mg}$ soit 1 comprimé ; (4) pour tous au-delà de 12 ans : $130 \mathrm{mg}$ de KI, soit deux comprimés. Les femmes enceintes doivent recevoir $130 \mathrm{mg}$ de KI (2 comprimés) pour protéger leur enfant. Les comprimés de KI doivent être pris en priorité par les enfants et les femmes enceintes. Cette prise est déconseillée aux sujets âgés de plus de 60 ans et aux adultes porteurs d'une pathologie thyroïdienne ou cardiovasculaire connue. Elle est selon toute vraisemblance inutile au-delà de 40 ans, car l'effet cancérigène des radiations sur la thyroïde n'est pas démontré à cet âge, et n'est indiquée que si la dose évaluée à la thyroïde risque de provoquer une hypothyroïdie en raison de son importance.

Cette prophylaxie par le $\mathrm{KI}$ a très peu d'effets secondaires. Des effets secondaires non spécifiques peuvent être observés, tels que nausées, vomissements, diarrhées, gastralgies, goût métallique dans la bouche. 


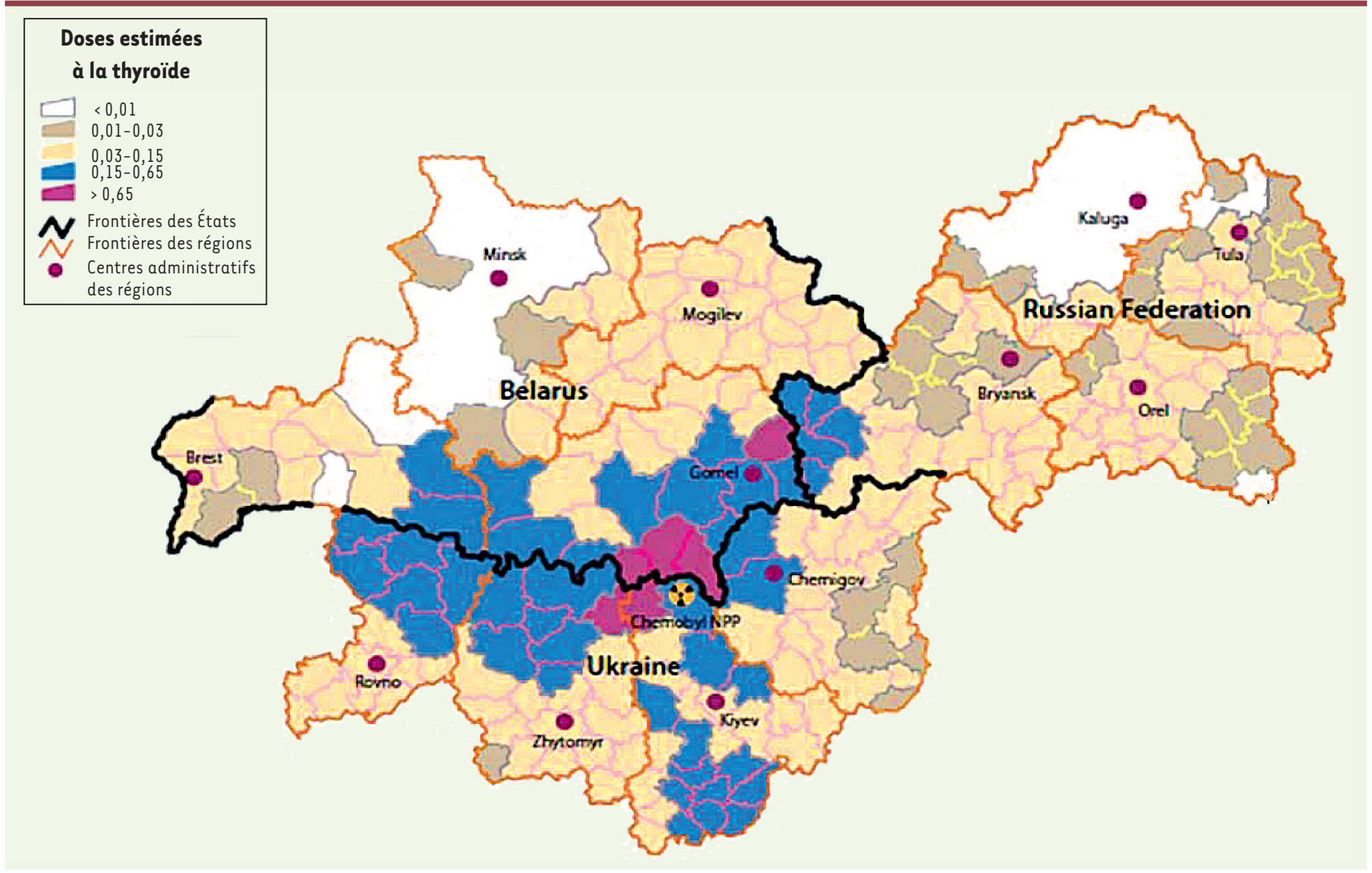

Figure 1. Distribution spatiale des doses estimées à la thyroïde chez les enfants et les adolescents après l'accident de Tchernobyl en Ukraine, en Biélorussie et en Russie. Données extraites du rapport UNSCEAR [1].

En Pologne, 18 millions de doses d'iode stable ont été distribuées et seuls trois cas de bronchospasmes spontanément réversibles ont été signalés [12]. Une hyper/hypothyroïdie peut survenir en cas de pathologie thyroïdienne préexistante, ce qui est exceptionnel chez le sujet jeune. La prise de $\mathrm{Kl}$ en fin de grossesse ou chez le nouveau-né peut provoquer une hypothyroïdie à la naissance, ce qui a été observé en Pologne, mais a été rapidement réversible et sans séquelle. Enfin, des hypersensibilités vraies sont exceptionnelles et peuvent survenir chez des patients atteints de pathologies très rares et bien caractérisées.

\section{Les mécanismes de la cancérogenèse thyroïdienne}

Le cancer de la thyroïde ne survient que chez un faible pourcentage des sujets ayant été exposés aux rayonnements ionisants. Une question se pose : le risque de cancer de la thyroïde est-il le même dans toute la population ou bien peut-on identifier une sous-population à risque? Plusieurs données épidémiologiques suggèrent que certaines circonstances prédisposent au développement d'une tumeur après exposition aux radiations ionisantes [7] : la présence de plusieurs tumeurs (tumeurs de la thyroïde, parathyroïde, tumeurs nerveuses et tumeurs des glandes salivaires) chez un même sujet après une exposition du cou aux radiations ionisantes ; les cas familiaux de cancers, et notamment de cancers de la thyroïde, qui se développent lorsque plusieurs membres d'une même famille ont été exposés à une irradiation externe ou ont été contaminés à la suite de l'accident de Tchernobyl [19-21]. Cette prédisposition pourrait être en rapport avec des anomalies dans la réparation de l'ADN. Les mécanismes par lesquels les radiations ionisantes provoquent l'apparition d'un cancer de la thyroïde sont probablement multiples et ils sont encore incomplètement connus. Ils associent des dommages à l'ADN (cassures simple et double-brins de l'ADN entraînant délétions et réarrangements) et l'induction d'une instabilité génétique qui va favoriser l'accumulation des lésions nécessaires à la transformation de la cellule thyroïdienne normale en cellule cancéreuse. Les cellules thyroïdiennes se multiplient pendant la croissance, surtout avant l'âge de cinq ans, ce qui permet l'accumulation des lésions génétiques, puis le taux de mitoses diminue avec l'âge pour devenir très faible à l'âge adulte. Ceci peut expliquer que la sensibilité aux effets cancérigènes des radiations soit maximale après la naissance, puis diminue avec l'âge, d'un facteur deux entre 5 et 10 ans, d'un facteur cinq entre 10 et 15 ans, pour n'être plus significative audelà de 15 à 20 ans $[7,8,21]$.

Pendant l'interphase des cellules thyroïdiennes, la proximité spatiale des gènes RET et CCDC6 (coiled-coil domain containing 6 ex $\mathrm{H} 4$ ) et $\varepsilon L E I$ explique la survenue de 
réarrangements intrachromosomiques RET/PTC (papillary thyroid carcinoma) 1 et RET/PTC3 après irradiation, via plusieurs étapes successives : cassures double-brin des deux gènes, inversion puis recombinaison.

Les radiations ionisantes engendrent la formation de radicaux libres oxygénés (ROS) résultant pour une grande part de la radiolyse de l'eau. Ces radicaux libres peuvent induire des cassures double-brin de I'ADN, et participent ainsi à la formation des réarrangements chromosomiques RET/PTC [22].

\section{Comment distinguer une tumeur radio-induite d'une tumeur spontanée}

Dans la plupart des pays occidentaux, on assiste depuis le début des années 1970 à une augmentation du nombre de cancers de la thyroïde diagnostiqués, liée essentiellement à un meilleur dépistage [13]. L'accident de Tchernobyl étant survenu à une période ou l'incidence était en augmentation, un rapprochement a été fait, bien que les doses d'irradiation reçues par la thyroïde aient été faibles en dehors des régions fortement contaminées de Biélorussie, d'Ukraine et de Russie. II s'agissait au maximum de quelques mSv chez les enfants vivant à l'époque dans l'est de la France, ce qui est de l'ordre de grandeur de l'irradiation naturelle annuelle qui est de 2,4 mSv (Tableau II).

Les calculs faits à partir du concept de dose collective (somme des doses individuelles, même minimes, reçues par une population importante) n'ont pas de sens biologique. La relation entre la dose et l'effet est linéaire pour des doses > $100 \mathrm{mSv}$ mais, pour des doses < $100 \mathrm{mSv}$, les mécanismes de réparation des lésions de l'ADN sont plus efficaces. Pour ces faibles niveaux de doses, l'effet est moins important que ce qui pourrait être déduit des effets des doses plus élevées. II n'est donc pas possible d'extrapoler les effets potentiels des doses faibles en raisonnant à partir de la dose collective et des effets des doses «fortes ». La possibilité de distinction fiable entre tumeurs radio-induites et tumeurs spontanées permettrait d'apporter des réponses aux questions posées sur les causes éventuelles de ce cancer et permettrait aussi d'améliorer la fiabilité des études épidémiologiques, notamment pour les faibles doses.

\section{Caractéristiques épidémiologiques et histocliniques}

Cette distinction se fonde d'abord sur des données épidémiologiques, car le risque de développer une tumeur de la thyroïde radio-induite n'est démontré que chez les personnes ayant reçu pendant l'enfance (avant l'âge de 15-20 ans) des doses d'irradiation au niveau de la thyroïde $>100 \mathrm{mSv}$, et seules les tumeurs apparaissant plus de 5 ans après l'exposition sont considérées comme radio-induites. Un facteur supplémentaire de suspicion est l'existence dans la famille d'une prédisposition à développer un cancer de la thyroïde (ou un autre cancer) après irradiation.

Sur le plan histologique, plus de $80 \%$ des cancers thyroïdiens en apparence spontanés ou survenus après irradiation ont un phénotype papillaire ${ }^{1}$. Parmi les cancers papillaires survenus précocement après

\footnotetext{
${ }^{1}$ Les cancers différenciés de la thyroïde développés à partir des thyrocytes sont d'histologie folliculaire, reproduisant à des degrés divers l'architecture thyroïdienne normale ou papillaire, avec des papilles et des cellules ayant des anomalies nucléaires caractéristiques; parmi ces cancers papillaires, on distingue la forme classique et plusieurs variants.
}

l'accident de Tchernobyl, le plus souvent chez de jeunes enfants, les formes solides ou folliculaires étaient fréquentes avec comme caractéristiques d'être volumineuses, étendues localement et compliquées de métastases ganglionnaires multiples et, fréquemment, de métastases pulmonaires. Les cancers survenus plus tardivement, chez des sujets souvent plus âgés, étaient des formes papillaires classiques, de volume plus faible, moins étendues et de progression plus lente.

Le pronostic des cancers radio-induits et des cancers en apparence spontanés est identique et est globalement favorable. Ainsi, parmi les 7000 patients atteints d'un cancer de la thyroïde considéré comme une conséquence directe de l'accident de Tchernobyl, environ 20 en sont décédés [1]. Toutefois, les cancers de la thyroïde des sujets jeunes qui ne sont pas guéris restent stables ou progressent très lentement et peuvent provoquer le décès de nombreuses années après leur découverte. Ils doivent donc être traités puis surveillés avec beaucoup de rigueur. La présentation et l'histoire naturelle des cancers spontanés et des cancers radioinduits survenus au même âge sont identiques, et les variations des formes histologiques et cliniques qui sont observées s'expliquent plus par l'âge de survenue du cancer que par son caractère radio-induit ou non $[7,21]$.

\section{Caractéristiques moléculaires}

Dans les cancers papillaires survenus après irradiation, les réarrangements intrachromosomiques RET/ PTC sont fréquents. Le réarrangement RET/PTC3 est plus fréquent dans les formes solides agressives, apparues chez des sujets jeunes rapidement après l'accident. Le réarrangement $R E T / P T C I$ est plus fréquent dans les formes classiques de cancer papillaire, apparues plus tardivement. De nombreux réarrangements RET/PTC qui diffèrent, soit par le gène partenaire, soit par le point de cassure, ont été décrits dans les cancers de la thyroïde survenus après l'accident de Tchernobyl, de même que des réarrangements impliquant le gène BRAF ( $V$-raf murine sarcoma viral oncogene homolog B1) [1, 21]. À l'inverse, les mutations ponctuelles de BRAF sont peu fréquentes dans les cancers papillaires survenus après exposition aux radiations.

En fait, les réarrangements $R E T / P T C$ sont également fréquents et les mutations BRAF rares dans les cancers papillaires de l'enfant qui surviennent en l'absence de toute exposition aux rayonnements ionisants. Chez l'adulte les réarrangements RET/PTC sont rares et les mutations BRAF fréquentes [23]. Ces données suggèrent que le spectre des mutations observées dans les cancers papillaires est lié à l'âge de survenue plus qu'à 
son étiologie. Ainsi, l'irradiation pourrait augmenter la fréquence d'un processus qui existe spontanément dans les cellules thyroïdiennes de l'enfant.

Récemment, une signature transcriptomique, qui identifie des expressions différentielles de gènes dans des tumeurs de la thyroïde selon qu'elles sont sporadiques ou se développent après une radiothérapie pendant l'enfance, a permis de discriminer ces deux groupes de tumeurs avec une sensibilité de 0,92 et une spécificité de 0,85 [24]. De plus, elle permet de distinguer les tumeurs sporadiques de celles survenues en Ukraine et en Biélorussie après l'accident de Tchernobyl [25]. Ceci confirme les résultats de certaines études antérieures [2628].

Ces études préliminaires laissent entrevoir l'existence de spécificités moléculaires dans les tumeurs de la thyroïde induites par les radiations. Ces résultats devront être confirmés sur de plus grandes séries de tumeurs, et d'autres techniques devront être utilisées, notamment l'expression des microARN, l'étude des pertes et des gains de matériel génétique, le séquençage en profondeur et l'étude des modifications épigénétiques.

Ces études transcriptomiques ont été réalisées sur des tumeurs secondaires à une exposition à des doses importantes de radiations. Elles ne sont pas applicables avant d'être validées au diagnostic du caractère radio-induit ou non d'une tumeur survenant chez une personne suspecte d'avoir été exposée à de faibles doses de radiations. De plus, la signification biologique de cette signature n'est pas claire. Elle peut être la marque d'une exposition antérieure aux radiations ionisantes (et alors sans conséquence sur la cancérogenèse), ou refléter le mécanisme de la cancérogenèse induite par les radiations ionisantes.

\section{Conclusion}

Le bilan sanitaire de l'accident de Tchernobyl a permis de mieux préciser les mesures à prendre pour protéger les populations en cas d'accident, notamment la prédistribution de comprimés d'iodure de potassium (KI).

En cas d'accident nucléaire, l'information et la communication jouent un rôle essentiel dans la diffusion des consignes des autorités compétentes visant à protéger les populations et éviter les phénomènes de panique et les fausses croyances. Comparé à l'accident de Tchernobyl, l'accident de Fukushima a montré une avancée significative dans la diffusion de l'information et les actions entreprises dans les 12 premières heures pour protéger les populations. La communication doit s'adresser également à l'ensemble de la population, qui a ses propres questionnements, et, dans ce domaine, des progrès sont nécessaires. La place de l'expertise pour établir une relation de confiance avec l'opinion (sûreté nucléaire, risques liés aux radiations, etc.) et pour aider le politique dans la prise de décision, reste aujourd'hui difficile à préciser dans un contexte général de défiance de la population vis-àvis des autorités. Par exemple, à Fukushima, les résultats des mesures réalisées sur les thyroïdes des enfants non évacués sont rassurants (doses à la thyroïde de 1 à 2 mSv au maximum) mais n'ont été communiqués que plusieurs semaines après l'accident.

\section{Annexe - Gestion sanitaire des travailleurs et de la population japonaise après l'accident de Fukushima}

Le tremblement de terre et le tsunami qui ont touché le nord-est du Japon le 11 mars 2011 ont eu pour conséquence immédiate 22626 morts ou disparus, dont 2 travailleurs sur le site de Fukushima.

\section{Situation radiologique des intervenants sur le site de} Fukushima

Plus de 20000 intervenants ont travaillé sur les deux sites de Fukushima depuis l'accident. Les conditions d'intervention ont été très difficiles, en particulier pendant les premières semaines, et on dénombre 25 blessés et 2 décès (malaise cardiaque, chute de grue).

En date du 31 décembre 2011, le bilan des expositions aux radiations des intervenants publié par TEPCO (Tokyo electric power company) était : 118 intervenants exposés à des doses «corps entier » comprises entre 100 et $150 \mathrm{mSv}, 22$ entre 150 et $250 \mathrm{mSv}$, et 6 à des doses supérieures à $250 \mathrm{mSv}$ (309-678 mSv). Aucune exposition à des doses supérieures au Sievert n'a été constatée, et aucun syndrome d'irradiation aiguë n'est survenu. Peu d'informations sont disponibles sur les expositions des secours externes.

L'évaluation des doses liées à la contamination interne n'a été réalisée que six semaines après l'accident, et son estimation a été difficile. Pour deux intervenants, les doses internes sont supérieures à $500 \mathrm{mSv}$. En termes de conséquences tardives, le faible effectif des personnels dont l'exposition a dépassé 100 mSv ne laisse pas prévoir d'augmentation décelable du nombre de cancers. Rappelons que selon la publication 103 de la CIPR (commission internationale de protection radiologique), l'incidence des cancers radio-induits est estimée à $4 \%$ par Sv (1000 mSievert).

\section{Conséquences pour la population}

Le principe de gestion du risque radiologique pour la population retenu par le gouvernement japonais a été de prendre en considération, dès le premier jour de l'accident, le seuil le plus bas recommandé pour les situations d'urgence par la CIPR, soit une limite maximale de $20 \mathrm{mSv}$ par an, et ce afin de minimiser le risque sanitaire. Ceci a conduit au déplacement de 85000 personnes, engagé dès le premier jour de l'accident, puis à la décision complémentaire, le 22 avril, d'évacuation au-delà du périmètre initial d'évacuation des 20 à $30 \mathrm{~km}$, dans une zone située dans le nord ouest où les retombées ont eu lieu après le 15 mars. 
Des conséquences affectant la santé publique sont liées au stress post-accidentel après la catastrophe naturelle et l'accident nucléaire, aux carences de la communication, aux mesures d'évacuation et aux conditions d'hébergement précaire. II peut en résulter, comme après l'accident de Tchernobyl, une morbidité importante comportant des syndromes dépressifs, des suicides, des addictions (tabac, alcool) sources elles-mêmes de pathologies, ainsi que l'aggravation de pathologies existantes.

Il est difficile d'évaluer avec précision le risque de cancer de la thyroïde pour les enfants exposés. Néanmoins, les décisions mises en place rapidement (évacuation, information et gestion de l'eau et des denrées), ainsi que les résultats des mesures réalisées sur la thyroïde, sont rassurants en termes d'impact sanitaire, avec des doses au niveau de la thyroïde de 1 à $2 \mathrm{mSv}$, et qui ne dépassent pas $10 \mathrm{mSv}$. Les autorités ont annoncé la mise en place d'un suivi pour les 360000 personnes âgées de 18 ans ou moins qui se trouvaient dans la préfecture de Fukushima au moment de l'accident.

Les conséquences environnementales dues aux rejets de césium sont importantes, environ $500 \mathrm{~km}^{2}$ devront être décontaminés, avec notamment l'existence de points chauds. Les actions engagées par les autorités japonaises ont pour but de permettre une levée partielle de l'évacuation début 2012 qui s'échelonnera sur plusieurs années, en dehors des zones trop durablement contaminées dans lesquelles l'ordre d'évacuation devra être maintenu.

L'objectif de la gestion à long terme de la contamination des territoires, due au seul césium 137, est de limiter l'exposition annuelle pour la population à moins de $20 \mathrm{mSv}$, pour revenir à moins de $1 \mathrm{mSv}$ au bout de quelques années et éviter ainsi tout impact sanitaire (le principe est que la dose cumulée sur plusieurs années ne dépasse pas 100 mSv). La qualité et la rapidité de la réhabilitation des territoires contaminés conditionneront des aspects essentiels de l'après-crise, aussi bien en termes d'impact sanitaire de l'accident que de jugement de la population japonaise vis-à-vis de la gestion d'un accident nucléaire.

Le gouvernement japonais a engagé le 11 novembre 2011 un plan très ambitieux et coûteux de décontamination et de gestion des déchets pour les territoires contaminés, pour permettre le retour à terme des populations dans la zone des $20 \mathrm{~km}$. Ce plan a pour objectif de décontaminer le territoire jusqu'à atteindre un niveau de dose de $1 \mathrm{mSv}$ par an, en privilégiant l'enlèvement de la couche superficielle de terre contaminée et la technique de labourage profond. Si en 2011, les premières mesures avaient privilégié la décontamination des écoles et des habitations, les mesures actuelles sont beaucoup plus globales et concernent de façon prioritaire uniquement les territoires où les doses estimées à l'année, pour un habitant, sont inférieures à $50 \mathrm{mSv}$ avec deux zones:

- Zone d'exposition de 1 à $20 \mathrm{mSv} / \mathrm{an}$, appelée «zone de préparation au retour ». Cette zone, qui représente près du tiers des territoires évacués, sera décontaminée en priorité, sous l'autorité des municipalités, pour s'approcher le plus possible d'une exposition de $1 \mathrm{mSv}$ / an. Des premiers retours de population (ville de Kawauchi) en limite de la zone d'exclusion ont été proposés mais, à l'instar de la région de Sendai, non touchée par les rejets radioactifs mais très marquée par les traces du passage du tsunami, nombreux sont ceux qui hésitent ou ont pris la décision de ne plus revenir.

- Zone d'exposition de 20 à $50 \mathrm{mSv} / \mathrm{an}$, représentant plus de la moitié des territoires évacués appelée «zone d'habitat limité ». Les opérations de décontamination, sous l'autorité du gouvernement, seront plus longues, et pourront durer quelques années et générer de nombreux déchets, et les autorisations de retour ne seront envisagées que lorsque l'exposition sera revenue à des niveaux proches de $1 \mathrm{mSv} / \mathrm{an}$.

Une forte contamination radioactive du milieu marin a été provoquée par le déversement direct d'eaux contaminées depuis la centrale et, plus faiblement, par les retombées des rejets radioactifs. L'IRSN (institut de radioprotection et de sûreté nucléaires) a estimé la quantité totale de césium 137 rejetée directement en mer du 21 mars jusqu'à mi-juillet 2011 à 27,1015 Bq. La localisation du site de Fukushima a permis une large dispersion des radionucléides, puisqu'un des courants les plus importants de l'océan Pacifique y circule, qui a éloigné les eaux contaminées vers le large. Les concentrations radioactives dans l'eau de mer sont normales depuis octobre 2011, sauf dans l'eau de mer qui borde le littoral proche de la centrale accidentée où une pollution significative pourrait persister dans le temps, entretenue par les apports continus de substances radioactives transportées vers la mer par le ruissellement des eaux de surface sur des sols contaminés. Les mesures récentes montrent la persistance d'une contamination des espèces marines (poissons principalement) pêchées sur les côtes de la préfecture de Fukushima. $\diamond$

\section{SUMMARY}

Nuclear-power-plant accidents: thyroid cancer incidence and radiation-related health effects from the Chernobyl accident

Following the Chernobyl accident, enormous amounts of radioisotopes were released in the atmosphere and have contaminated surrounding populations in the absence of rapid protective countermeasures. The highest radiation doses were delivered to the thyroid gland, and the only direct consequence of radiation exposure observed among contaminated population is the increased incidence of thyroid cancers among subjects who were children in 1986 and who lived at that time in Belarus, Ukraine or Russia. $\diamond$

\section{LIENS D'INTÉRÊT}

M. Schlumberger conseille EDF pour les problèmes sanitaires associés à l'exposition aux rayonnements ionisants. B. Le Guen est Directeur délégué radioprotection-sécurité d'EDF. 


\section{RÉFÉRENCES}

1. United Nations scientific committee on the effects of atomic radiation. Sources and effects of ionizing radiation. Health effects due to radiation from the Chernobyl accident. In : UNSCEAR 2008, vol II. New York : United Nations, 2011 : annexe D.

2. Christodouleas JP, Robert D, Forrest RD, et al. Short-term and long-term health risks of nuclearpower-plant accidents. N Engl J Med 2011 ; $364: 2334-41$.

3. Loevinger R, Budinger TF, Watson દદ. MIRD Primer. Reston, VA: Society of Nuclear Medicine, 1999.

4. Preston DL, Ron $\varepsilon$, Tokuoka S, et al. Solid cancer incidence in atomic bomb survivors: 1958-1998. Radiat Res 2007 ; 168 : 1-64.

5. Green DM, Whitton JA, Stovall M, et al. Pregnancy outcome of female survivors of childhood cancer: a report from the Childhood Cancer Survivor Study. Am J Obstet Gynecol 2002 ; 187 : 1070-80.

6. Garsi JP, Schlumberger M, Rubino C, et al. Therapeutic administration of ${ }^{131} 1$ for differentiated thyroid cancer, radiation dose to ovaries and outcome of pregnancies. J Nucl Med 2008 ; 49 : $845-52$.

7. Sinnott B, Ron $\varepsilon$, Schneider AB. Exposing the thyroid to radiation: a review of its current extent, risks, and implications. Endocr Rev 2010 ; 31 : 756-73.

8. Ron $\varepsilon$, Lubin JH, Shore RE, et al. Thyroid cancer after exposure to external radiation: a pooled analysis of seven studies. Radiat Res 1995 ; 141 : 259-77.

9. Simon SL, Bouville A, Land CE, Beck HL. Radiation doses and cancer risks in the Marshall Islands associated with exposure to radioactive fallout from Bikini and Enewetak nuclear weapons tests: summary. Health Phys $2010 ; 99: 105-23$.

10. Dickman PW, Holm LE, Lundell G, et al. Thyroid cancer risk after thyroid examination with 131l: a population-based cohort study in Sweden. Int J Cancer $2003 ; 106: 580-7$.

11. Holm LE, Hall P, Wiklund $K$, et al. Cancer risk after iodine-131 therapy for hyperthyroidism. J Natl Cancer Inst $1991 ; 83: 1072-7$.

12. Nauman J, Wolff J. lodide prophylaxis in Poland after the Chernobyl reactor accident: benefits and risks. Am J Med 1993 ; $94: 524-32$.

13. Colonna M, Guizard AV, Schvartz C, et al. A time trend analysis of papillary and follicular cancers as a function of tumour size: a study of data from six cancer registries in France (1983-2000). Eur J Cancer $2007 ; 43: 891-900$.

14. Renehan AG, Tyson M, Egger $M$, et al. Body-mass index and incidence of cancer: a systematic review and meta-analysis of prospective observational studies. Lancet $2008 ; 371: 569-78$.

15. Sermage-Faure C, Laurier D, Goujon-Bellec $S$, et al. Childhood leukemia around French nuclear power plants. The geocap study, 2002-2007. Int J Cancer 2012; doi: 10.1002/ijc.27425.

16. Zanzonico PB, Becker DV. Effects of time of administration and dietary iodine levels on potassium iodide (KI) blockade of thyroid irradiation by 131 from radioactive fallout. Health Phys 2000 ; $78: 660-7$.
17. Loi sur la transparence et la sécurité en matière nucléaire. Loi n 2006-686 du 13 juin 2006. $J 014$ juin 2006 ; 136.

18. Le Guen B, Stricker L, Schlumberger M. Prophylaxis of thyroid exposure in case of nuclear power plant accident. Experience with the French distribution of KI pills. Nat Clin Pract Endocrinol Metab $2007 ; 3: 611$.

19. Perkel VS, Gail MH, Lubin J, et al. Radiation-induced thyroid neoplasms: evidence for familial susceptibility factors. J Clin Endocrinol Metab 1988 ; $66: 1316-22$.

20. Adjadj $\varepsilon$, Schlumberger M, de Vathaire F. Germ-line DNA polymorphisms and susceptibility to differentiated thyroid cancer. Lancet Oncol 2009; 10 181-90.

21. Williams D. Radiation carcinogenesis: lessons from Chernobyl. Oncogene $2009 ; 27:$ S9-18.

22. Ameziane- $\varepsilon l-H a s s a n i \mathrm{R}$, Boufraqech $M$, Lagente-Chevallier 0 , et al. Role of $\mathrm{H}_{2} \mathrm{O}_{2}$ in RET/PTCl chromosomal rearrangement produced by ionizing radiation in human thyroid cells. Cancer Res $2010 ; 70: 4123-32$.

23. Sassolas G, Hafdi-Nejjari Z, Ferraro A, et al. Oncogenic alterations in papillary thyroid cancers of young patients. Thyroid $2012 ; 22: 17-26$.

24. Ory C, Ugolin N, Levalois C, et al. Gene expression signature discriminates sporadic from post-radiotherapy-induced thyroid tumors. Endocr Relat Cancer $2011 ; 18: 193-206$.

25. Ory C, Ugolin N, Schlumberger M, et al. Discriminating gene expression signature of radiation-induced thyroid tumors after either external exposure or internal contamination. Genes $2012 ; 3: 19-34$

26. Detours V, Delys L, Libert F, et al. Genome-wide gene expression profiling suggests distinct radiation susceptibilities in sporadic and post-Chernobyl papillary thyroid cancers. BrJ Cancer 2007 ; $97: 818-25$.

27. Port $M$, Boltze $C$, Wang $Y$, et al. A radiation-induced gene signature distinguishes post-Chernobyl from sporadic papillary thyroid cancers. Radiat Res 2007 ; 168 : 639-49.

28. Stein L, Rothschild J, Luce J, et al. Copy number and gene expression alterations in radiation-induced papillary thyroid carcinoma from Chernobyl pediatric patients. Thyroid $2010 ; 20: 475-87$.

\section{TIRÉS À PART}

M. Schlumberger

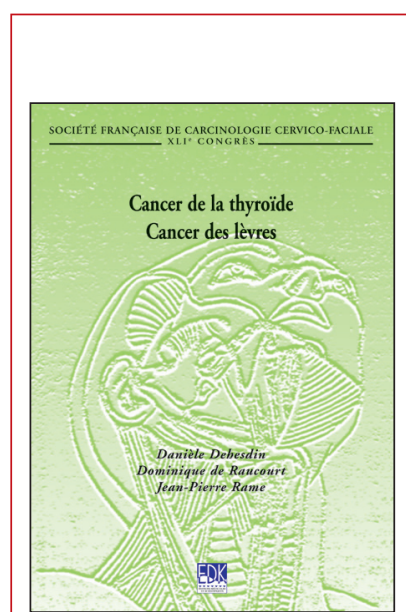

ISBN : 978-2-8425-4137-8 264 pages

\section{Bon de commande}

À retourner à EDK, 25, rue Daviel - 75013 Paris

Tél. : 0158101905 - Fax : 0143293262 - E-mail : edk@edk.fr

NOM :

Prénom :

Adresse :

Code postal :

Ville :

Pays :

Fonction :

Je souhaite recevoir l'ouvrage Cancer de la thyroïde - Cancers des lèvres : $35 €+3 €$ de port $=\mathbf{3 8} € \mathbf{T T C}$

en ................ exemplaire, soit un total de $€$

$\square$ Par chèque, à l'ordre de $\mathbf{E} \mathbf{D} \mathbf{K}$

Par carte bancaire : $\quad \square$ Visa $\square$ Eurocard/Mastercard

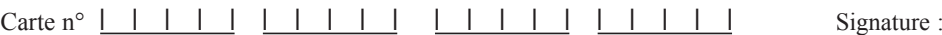

Date d'expiration: $\quad|\quad| \quad|\quad| \quad \mid$

$\mathrm{N}^{\circ}$ de contrôle au dos de la carte : $\quad$ | $1 \quad 1 \quad$ 